\title{
Defining and Model Checking Abstractions of Complex Railway Models Using CSP $\| \mathrm{B}$
}

\author{
Faron Moller ${ }^{1}$, Hoang Nga Nguyen ${ }^{1}$, Markus Roggenbach ${ }^{1}$, \\ Steve Schneider ${ }^{2}$, and Helen Treharne ${ }^{2}$ \\ 1 Swansea University, Wales, UK \\ 2 University of Surrey, England, UK
}

\begin{abstract}
The safety analysis of interlocking railway systems involves verifying collision and derailment freedom. In this paper we propose a structured way of refining track plans, in order to expand track segments so that they form collections of track segments. We show how the abstract model can be model checked to ensure the safety properties, which must also hold in the corresponding concrete track plan, so that we will never need to model check the concrete track plan directly. We also identify the minimal number of trains that needs to be considered as part of the model checking, and we demonstrate the practicality of the approach on various scenarios.
\end{abstract}

\section{Introduction}

Formal verification of railway control software has been identified as one of the "Grand Challenges" of Computer Science [11. As is typical with Formal Methods, this challenge comes in two parts: the first addresses the question of whether the mathematical models considered are legitimate representations of the physical systems of concern. The modelling of the systems, as well as of proof obligations, needs to be faithful. The second part is the question of how to utilize available technologies, for example model checking or theorem proving. Whichever verification process is adopted, it needs to be both effective and efficient.

In 1312 we proposed a new modelling approach for railway interlockings. We use CSP $\| \mathrm{B}$ [15], which combines event-based with state-based modelling. This reflects the double nature of railway systems, which involves events such as train movements and, in the interlocking, state based reasoning. In this sense, CSP $\| \mathrm{B}$ offers the means for the natural modelling approach we strive for: the formal models are close to the domain models. To the domain expert, this provides traceability and ease of understanding. This addresses the first of the above stated challenges: faithful modelling.

In this paper, we address the question of how to effectively and efficiently verify various safety properties within our CSP $\|$ B models. To this end we develop a set of abstraction techniques for railway verification that allow the transformation of complex CSP $\| \mathrm{B}$ models into less involved ones, prove that they are 
correct, and demonstrate that they allow one to verify a variety of railway systems via model checking. The first set of abstractions reduces the number of trains that need to be considered in order to prove safety for an unbounded number of trains. Their correctness proof involves slicing of event traces. Essentially, these abstractions provide us with finite state models. The second set of abstractions simplifies the underlying track topology. Here, the correctness proof utilizes event abstraction specific to our application domain similar to the ones suggested by Winter in [17]. These abstractions make model checking faster.

Outline. We first introduce our modelling language CSP $\|$ B. In Section 3 we summarise our generic railway modelling approach using CSP $\| \mathrm{B}$, as described in 1312 . In Section 4, we present our first set of abstraction techniques based on event traces. Then in Section 5 we present our data abstraction techniques. The application of the abstraction results is presented via a set of example scenarios in Section [6. In Section 7 we put our work in the context of related approaches.

\section{Background to CSP $\| \mathrm{B}$}

The CSP $\|$ B approach allows us to specify communicating systems using a combination of the B-Method [4] and the process algebra CSP (Communicating Sequential Processes) 9]. The overall specification of a combined communicating system comprises two separate specifications: one given by a number of CSP process descriptions and the other by a collection of B machines. Our aim when using B and CSP is to factor out as much of the "data-rich" aspects of a system as possible into B machines. The B machines in our CSP $\| \mathrm{B}$ approach are classical B machines, which are components containing state and operations on that state. The CSP $\|$ B theory [15] allows us to combine a number of CSP processes $P s$ in parallel with machines $M s$ to produce $P s \| M s$ which is the parallel combination of all the controllers and all the underlying machines. Such a parallel composition is meaningful because a $\mathrm{B}$ machine is itself interpretable as a CSP process whose event-traces are the possible execution sequences of its operations. The invoking of an operation of a B machine outside its precondition within such a trace is defined as divergence [14. Therefore, our notion of consistency is that a combined communicating system Ps $\| M s$ is divergence-free and also deadlock-free.

A B MACHINE clause declares a machine and gives it a name. The VARIABLES of a B machine define its state. The INVARIANT of a B machine gives the type of the variables, and more generally it also contains any other constraints on the allowable machine states. There is an INITIALISATION which determines the initial state of the machine. The machine consists of a collection of OPERATIONS that query and modify the state. Besides this kind of machine we also define static B machines that provide only sets, constants and properties that do not change during the execution of the system. 
The language we use to describe the CSP processes for $\mathrm{B}$ machines is as follows:

$$
\begin{gathered}
P::=e ? x ! y \rightarrow P(x)\left|P_{1} \square P_{2}\right| P_{1} \sqcap P_{2} \mid \text { if } b \text { then } P_{1} \text { else } P_{2} \text { end | } \\
N(\exp ) \quad\left|\quad P _ { 1 } \left\|P _ { 2 } \left|P _ { 1 } \left\|_{B} P_{2}\left|P_{1} \|\right| P_{2}\right.\right.\right.\right.
\end{gathered}
$$

The process $e ? x ! y \rightarrow P(x)$ defines a channel communication where $x$ represents all data variables on a channel, and $y$ represents values being passed along a channel. Channel $e$ is referred to as a machine channel as there is a corresponding operation in the controlled B machine with the signature $x \longleftarrow e(y)$. Therefore the input of the $\mathrm{B}$ operation $y$ corresponds to the output from the CSP, and the output $x$ of the B operation to the CSP input. Here we have simplified the communication to have one output and one input but in general there can be any number of inputs and outputs. The other CSP operators have the usual CSP semantics.

For reasoning of $\mathrm{CSP} \| \mathrm{B}$ models we require the following notation.

- Since a B machine is interpretable as a CSP process, the various CSP refinements also apply to CSP $\|$ B. In this paper we focus on trace refinement where $P \sqsubseteq_{T} Q$ if $\operatorname{traces}(Q) \subseteq \operatorname{traces}(P)$. This refinement preserves safety properties, such as collision freedom or derailment freedom as we shall discuss in Section 3 .

- Furthermore, we apply CSP renaming $f(P)$ and CSP hiding $P \backslash A$ to CSP processes, B machines and to CSP $\|$ B models, which all semantically represent sets of traces. Given a set of traces $T, f(T)$ represents the set of all traces $\operatorname{tr} \in T$ where the events are replaced point-wise by the function $f$; $T \backslash A$ to represent the set of all traces $\operatorname{tr} \in T$ where the events from the set $A$ are removed from $t r$.

- A system run $\sigma$ (of a CSP $\| \mathrm{B}$ model) of length $n \geq 0$ is a finite sequence

$$
\sigma=\left\langle s_{0}, e_{0}, s_{1}, e_{1}, \ldots, e_{n-1}, s_{n}\right\rangle
$$

where the $s_{i}, i=0 \ldots n$, are states of the $B$ machine, and the $e_{i}, 1 \leq i \leq$ $n-1$, are events - either controlled by CSP and enabled in B when called, or B events. Here we assume that $s_{0}$ is a state after initialisation. Given a system run $\sigma$, we can extract its trace of events:

$$
\operatorname{events}(\sigma)=\left\langle e_{0}, \ldots, e_{n-1}\right\rangle
$$

\section{Modelling and Safety Verification of Railway Systems Using CSP $\| \mathrm{B}$}

Together with railway engineers we developed a common view on the information flow in railways. In physical terms a railway consists of, at least, four different components. These components are shown in Figure 1. The Controller selects and releases routes for trains. The Interlocking serves as a safety mechanism 
with regards to the Controller and, in addition, controls and monitors the Track equipment. The Track equipment consists of elements such as signals, points, and track circuits (logical names for tracks and points from the track plan as discussed above; in the railway domain, tracks and track circuits are often confused): signals can show the aspects green or red; points can be in normal position (leading trains straight ahead) or in reverse position (leading trains to a different line) and track circuits detect if there is a train on a track. Finally, Trains have a driver who determines their behaviour. For the purposes of modelling, we make the assumption that track equipment reacts instantly and is free of defects. The information flow shown in Figure 1 is as follows: the controller sends a request message to the interlocking to which the interlocking responds; the interlocking sends signalling information to the trains; and the trains inform the interlocking about their movements. The interlocking serves as the system's clock: messages can be exchanged once per cycle.

In this paper, we study various track plans, one of which is a station illustrated in Figure 2(b). It depicts the scheme plan for the station, which comprises a track plan, a control table, and release tables. (We will discuss Figure 2(a) in Section 6).

The track plan provides the topological information of the station which consists of 16 tracks (e.g., the track $\left.c_{-} T A A\right)$, three signals (e.g., $S 1$ ), and two points (e.g., P1). Note that the tracks include entry and exit tracks on which trains can "appear" and "disappear". These two kinds of tracks are specially treated during

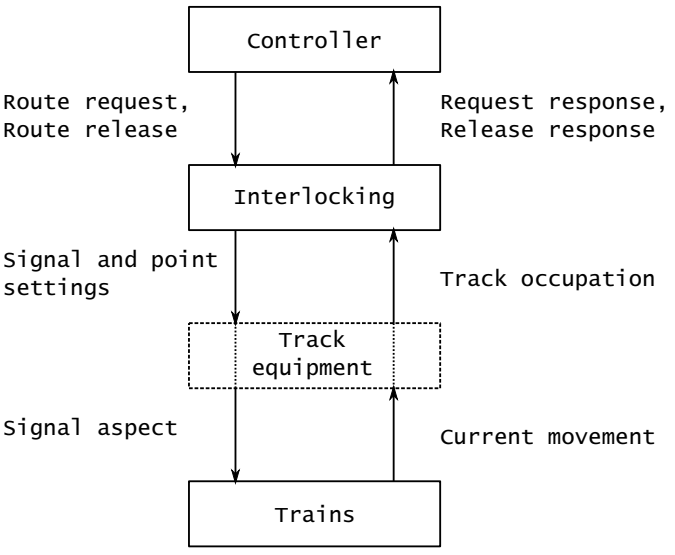

Fig. 1. Information flow verification.

An interlocking system gathers train locations, and sends out commands to control signal aspects and point positions. The control table determines how the station interlocking system sets signals and points. For each signal, there is one row describing the condition under which the signal can show proceed. There are two rows for signal $S 1$ : one for the main line (Route A1) and one for the side line (Route B1). A route comprises tracks and points between two signals. For example, signal S1 for the main line can only show proceed when point $\mathrm{P} 1$ is in normal (straight) position and tracks $c_{-} T A A, c_{-} T A B, c_{-} T A C, c_{-} T A D, c_{-} T A E$, $c_{-} T A F, c_{-} T A G$ are all clear. Here we assume that trains are equipped with an Automatic Train Protection system which prevents trains from moving over a red light and therefore, overlaps are not needed, e.g., the overlap for Route A1 would be $c_{-} T A H$. For further discussion on this see [10]. 


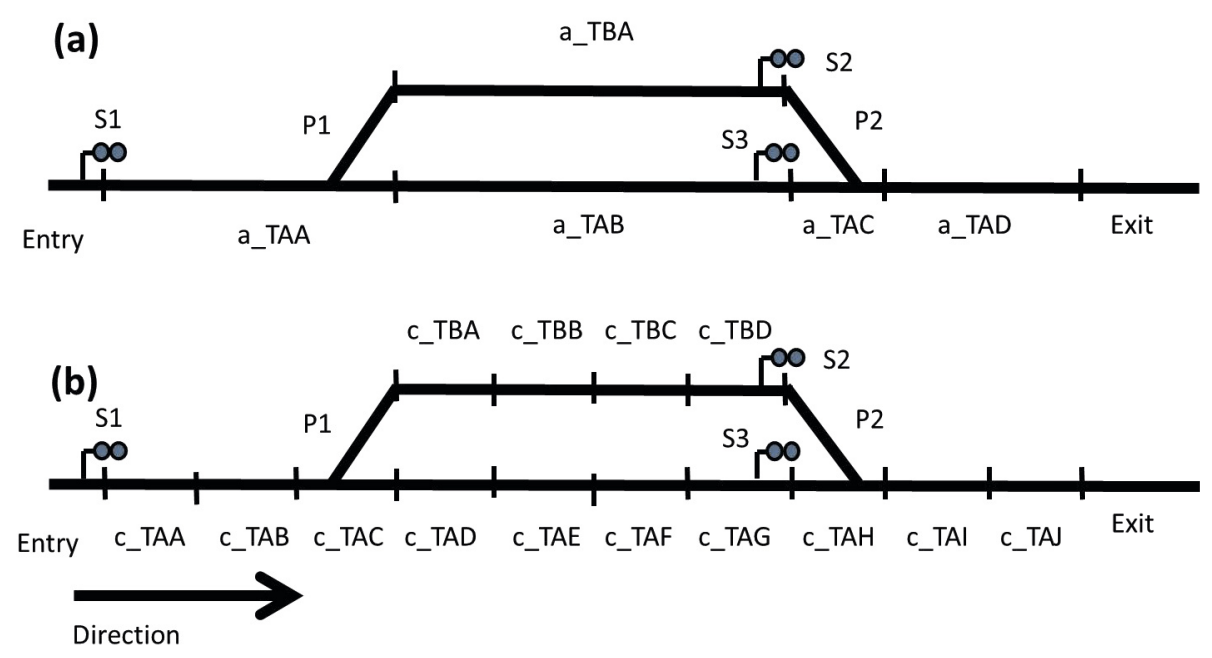

\section{Control table}

\begin{tabular}{|c|c|c|l|}
\hline Route & Normal & Reverse & Clear \\
\hline \hline A1 & P1 & & $\begin{array}{l}c_{-} T A A, c_{-} T A B, c_{-} T A C, c_{-} T A D, \\
c_{-} T A E, c_{-} T A F, c_{-} T A G\end{array}$ \\
\hline B1 & & P1 & $\begin{array}{l}c_{-} T A A, c_{-} T A B, c_{-} T A C, c_{-} T B A, \\
c_{-} T B B, c_{-} T B C, c_{-} T B D\end{array}$ \\
\hline A2 & P2 & & $c_{-} T A H, c_{-} T A I, c_{-} T A J$ \\
\hline B2 & & P2 & $c_{-} T A H, c_{-} T A I, c_{-} T A J$ \\
\hline
\end{tabular}

Release tables

\begin{tabular}{|c|c|}
\hline P1 & Occupied \\
\hline \hline $\mathrm{A} 1$ & $c_{-} T A D$ \\
\hline $\mathrm{B} 1$ & $c_{-} T B A$ \\
\hline
\end{tabular}

\begin{tabular}{|l|c|}
\hline P2 & Occupied \\
\hline \hline A2 & $c_{-} T A I$ \\
\hline B2 & $c_{-} T A I$ \\
\hline
\end{tabular}

Fig. 2. One Station - Abstract (a) and Concrete (b) Track Plan (Scenario 3 from Fig. 4)

The interlocking also allocates locks on points to particular route requests to keep them locked in position, and releases such locks when trains have passed. For example, the setting of Route A1 obtains a lock on point P1, and sets it to normal. The lock is released after the train has passed the point. Release tables store the relevant track.

In this setting, we consider two safety properties: collision-freedom excludes two trains occupying the same track; and no-derailment says that whenever a train enters a point, the point is set to cater for this; e.g., when a train travels from track $c_{-} T A G$ to track $c_{-} T A H$, point P2 is set so that it connects $c_{-} T A G$ and $c_{-} T A H$ (and not $c_{-} T B D$ and $c_{-} T A H$ ). The correct design for the control table and release tables is safety-critical: mistakes can lead to collision or derailment.

\subsection{CSP ||B Modelling of Railways}

In previous work [1213] we have demonstrated that CSP $\| \mathrm{B}$ caters for railways. It is possible to read the actual models together with railway engineers in order to validate them. This review demonstrates that the models can be clearly 
understood by railway engineers. Here, we refrain from elaborating on the modelling approach and refer the interested reader to [13] for the details. However, the concepts from the models central for verification (in Section 4 and Section (5), namely static and dynamic state representation and also train movements, are discussed below.

The static state information of a CSP $\| \mathrm{B}$ model is defined in context machines, i.e., machines that contain set and function definitions. For example, the names of all the track circuits is defined in a set called ALLTRACKS. The topology of the track plan is captured using a collection of relations that capture how the elements of the track plan are related. For example, next : TRACK $\leftrightarrow$ $T R A C K$ is a relation between tracks and possible successor tracks. Therefore, $\left(c_{-} T A C, a_{-} T A D\right)$ and $\left(c_{-} T A C, c_{-} T B A\right)$ are elements of the next relation within the one-station example in Figure 2 .

The Interlocking machine models the dynamics of the system. Its state evolves over time. It consists of the following variables: pos representing the position of all trains, nextd representing the current position of all points (and thus the dynamic relation between tracks and their successors), signalStatus representing the aspect of each signal, normalPoints representing the points which are in normal position, reversePoints representing the points which are in reverse position, and currentLocks representing the current semaphores on points.

In the CSP $\| \mathrm{B}$ models, a train $a$ can perform one of the following events: move.a.currp.newp represents a moving from track currp to track newp, nextSignal.a.aspect represents $a$ seeing the particular aspect (red or green) at the next signal, enter.a.p represents placing $a$ on an entry track $p$, and exit.a.p represents $a$ leaving the system. Trains that have left the system can be placed again on an entry track; we call this behaviour recurring trains. Note that in the situation where currp and newp are separated by a signal the event move.a.currp.newp is possible only if this signal shows green.

\section{Providing Finite State Models}

Our railway models are infinite state in nature. The reason for this is that we consider train identifiers explicitly. Therefore, it is essential to find bounds for the number of trains that we need to consider when analysing our models for safety. In this section we provide two methods: one tailored towards collision freedom, one designed for derailment freedom.

\subsection{Minimum Number of Trains for Verifying Collision}

The following theorem turns the question of whether a railway scheme plan is collision free into a finite state problem by reducing the - in principle - unbounded number of trains to be considered into a finite number:

Theorem 1. Let $S$ be a railway scheme plan with $r$ routes. $S$ is collision free iff all systems runs with $r+1$ recurring trains are collision free. 
Proof. We prove the "if" direction only, as the other direction trivially holds.

We first note that if there are two trains on a route then a collision can occur (as these two trains are not separated by a signal). Therefore, as long as there is no collision there will be at most $r$ trains on $S$. Assuming we have $r+1$ trains there will always be one train available to move onto an entry track. Thus, $r+1$ recurring trains are sufficient.

\subsection{Minimum Number of Trains for Verifying Derailment}

Regarding derailment, we obtain an even stronger result. The reduction argument, however, holds only for "reasonable" scheme-plans where the various tables are free of trivial mistakes with respect to the railway topology. Concretely, we say that a scheme plan is well-formed if the following conditions hold:

1. Release-Table condition. Locks of a route can only be released by a train movement on this route (e.g., in Figure 2, there is the lock $c_{-} T A D$ on P1 for route $A 1 ; c_{-} T A D$ appears in the clear column of the control table for the route $\mathrm{A} 1$ ).

2. Clear-Table condition. The clear table of a route contains at least the tracks of this route (e.g., in Figure 2 route A1 topologically goes from signal S1 to signal S3 and all tracks from $c_{-} T A A$ to $c_{-} T A G$ are in the clear column of the control table for the route A1).

3. Normal/Reverse-Table condition. The normal table or the reverse table of a route contain at least the points on this route (e.g., in Figure 2 route A1 topologically goes from signal S1 to signal S3, it includes the only point $\mathrm{P} 1$, and $\mathrm{P} 1$ is in the normal column of the control table for the route A1).

4. Route condition. Topologically different routes are distinguishable by point positions in the control table (e.g., in Figure 2 route A1 and route B1 are topologically different, point P1 is in the normal column of the control table for route $\mathrm{A} 1$, point $\mathrm{P} 1$ is in the reverse column of the control table for route B1).

5. Lock-Table condition. Routes with different lock tables are distinguishable by point positions in the control table (e.g., in Figure 2 route A1 and route $\mathrm{B} 1$ have different lock table entries, namely, $c_{-} T A D$ and $c_{-} T B A$ respectively, in the control table the position of $\mathrm{P} 1$ distinguishes them as seen above).

The scheme plan of Figure 2 is well-formed.

Note that there is exactly one condition per table (release table, clear table, normal/reverse table, lock table) plus one condition which links routes as defined topologically with the route definition in the tables. All five conditions are static and can easily be decided for a given scheme-plan. It is worthwhile to point out that well-formedness does not imply the property "no-derailment":

Observation 1. There exist well-formed scheme-plans with derailment.

For example, altering the scheme plan of Figure 2 by exchanging the position of point P2 for route A2 and route B2 leads to derailment as explained in Section 3 . This exchange, however, preserves well-formedness. 
Our modelling characterizes only implicitly, which routes which are set. Therefore, we the following theorem is helpful:

Theorem 2. For all system runs of a well-formed scheme-plans it holds: If a signal s shows green, then there exists a route $r$ with $\operatorname{signal}(r)=s$ which is set.

Then, we establish the following theorem which allows the reduction of the number of trains for proving derailment freedom:

Theorem 3. For any collision free system run on a well-formed scheme plan involving $k \geq 1$ trains Trains $=\left\{a_{1}, \ldots, a_{k}\right\}$ and a train $b$ which does not derail in this run, there exists a system run involving only the trains $\left\{a_{1}, \ldots, a_{k}\right\}$ with identical movements.

Proof. (Sketch) Let $\sigma$ be the system with trains in $\left\{a_{1}, \ldots, a_{k}, b\right\}$ where $b$ does not derail. We shall construct another run $\sigma^{\prime}$ which

- does not speak about $b$, which,

- however, preserves the movement of all trains $a_{i} \in$ Trains.

First, we define the set of all events $E(b)$ that are related with the train $b$ :

$$
\begin{aligned}
E(b):=\{e \in \sigma \mid & =\text { move.b.currp.newp } \\
e & =\text { nextSignal.b.aspect } \\
& =\text { enter.b.p } \\
e & =\text { exit.b.p }\}
\end{aligned}
$$

Intuitively, $\sigma^{\prime}$ is obtained from $\sigma$ by either discarding or replacing events in $E(b)$. In order to determine how to treat these events, it is necessary to understand how the train $b$ can influence the trains $a_{i} \in$ Trains: (i) $b$ might prevent a train $a \in$ Trains from moving (because a signal in front of $a$ shows red because $b$ uses a resource); (ii) $b$ might allow a train in Trains to move (a move from $b$ releases a lock, so that the signal in front of $a$ can change to green). When "taking away" $b$ from $\sigma$ our only concern is (ii): we wish to preserve moves. This insight leads to the definition of the following replacement function replace $_{b}$ concerning events (where $\epsilon$ stands for the empty word, i.e., for deletion of the event):

1. $\operatorname{replace}_{b}(e)=e$ if $e \notin E(b)$

2. replace $_{b}$ (move.b.currp.newp $)=$ release.r.bb if there exists a signal $s$ with currp $=$ homeSignal $(s)$. As move is only enabled if signal $s$ shows green, Theorem 2 guarantees that there exists a route $r$ which is set. Well-formedness of the scheme-plan guarantees uniqueness.

3. replace $_{b}(e)=\epsilon$ if $e$ is any of move.b.currp.newp, where currp $\neq$ homeSignal $(s)$ for any signal s, or nextSignal.b.aspect, or enter.b.p, or exit.b.p.

replace $_{b}$ keeps all events not related to $b(1$.), releases all locks related to $b$ at the earliest possible opportunity (2.), and deletes all other events related to $b$.

In order to show that the constructed $\sigma^{\prime}$ is a system run, we relate states in $\sigma$ with those in $\sigma^{\prime}$. Informally, a state $S$ in $\sigma$ is related to a state $T$ in $\sigma^{\prime}$, written 
as $S \geq_{b} T$, if (1) for all trains $a_{i}$ it holds that in $S$ and in $T$ (i) their positions are the same and (ii) they are offerred the same possibilities to move; and (2) $T$ does not speak about the train $b$. To capture these ideas formally, we define that $S \geq_{b} T$ if

1. Compared to $S, T$ just deletes the information regarding $b$. $T($ pos $)=S($ pos $) \backslash\{b \mapsto$ track $\mid$ track $\in T R A C K\}$

2. Track equipment is in the same state.

$$
\begin{array}{ll}
S(\text { next })=T(\text { next }) & S(\text { signalStatus })=T(\text { signalStatus }) \\
S(\text { normalPoints })=T(\text { normalPoints }) & S(\text { reversePoints })=T(\text { reversePoints })
\end{array}
$$

3. A route $r$ causes locks in $T$ only if it does so in $S$ :

$S($ currentLocks $[\{r\}])=\emptyset \Rightarrow T($ currentLocks $[\{r\}])=\emptyset$ and

$T($ currentLocks $[\{r\}]) \neq \emptyset \Rightarrow S($ currentLocks $[\{r\}])=T($ currentLocks $[\{r\}])$

Let $\sigma=\left\langle S_{0}, e_{0}, S_{1}, e_{1}, \ldots, e_{n-1}, S_{n}\right\rangle$, we obtain $\sigma^{\prime}$ in two steps. First, we define the sequence of events:

$$
\text { events }\left(\sigma^{\prime}\right)=\left\langle\text { replace }_{b}\left(e_{0}\right), \ldots, \text { replace }_{b}\left(e_{n-1}\right)\right\rangle
$$

Then, we replace in each step $\left\langle S_{i}, e_{i}, S_{i+1}\right\rangle$ of $\sigma$ the result state:

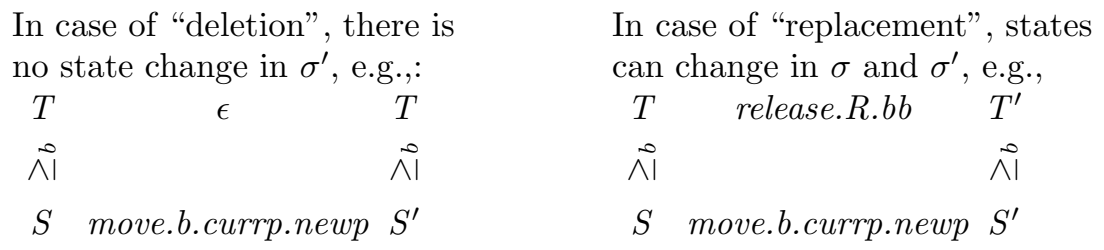

Finally, we prove that the so constructed $\sigma^{\prime}$ is indeed a system run by induction on the length of the system run $\sigma$. The base case is given by $S_{0} \geq_{b} S_{0}$ where $S_{0}$ is the initial state which has no trains in and there are no locks for points. In the induction step we show: (i) if an event $e$ is enabled in $S$ then replace $_{b}(e)$ is enabled in the corresponding state; (ii) $\geq_{b}$ is preserved under the execution under an event $e$ and its corresponding event replace ${ }_{b}(e)$. Both arguments rely on the fact that $\sigma$ is a system run, i.e., is a control flow allowed by the CSP processes.

The condition "collision free" on the system run $\sigma$ is required, as we "simulate" the movement of the train $b$ by a route release request. Routes can only be released if there is no train on the track $t$ directly in front of the corresponding signal. In the corresponding run $\sigma^{\prime}, b$ will not be on track $t$, as $b$ has been removed. There might, however, be another train $a$. We exclude this by the condition "collision freedom": if there was a train $a$ on the same track $t$ as train $b$, there would be a collision in $\sigma$.

Corollary 1. For collision free and well-formed scheme plans holds: if they are derailment free for one train, then they are derailment free for any number of trains. 


\section{$5 \quad$ Simplifying Scheme Plans}

In this section we prove, by topological argument, that it is sufficient to check a simple scheme plan for safety in order to establish safety for a complex scheme plan. The technical means for this is to establish a B refinement.

Let us consider an example in order to demonstrate the effect that the number of tracks per route has on model checking. Figure 3 shows three track plans. Track plan (a) has one track per route, track plan (b) has two tracks per route, and track plan (c) has four tracks per route. Below, we show how the state space grows in the number of tracks per
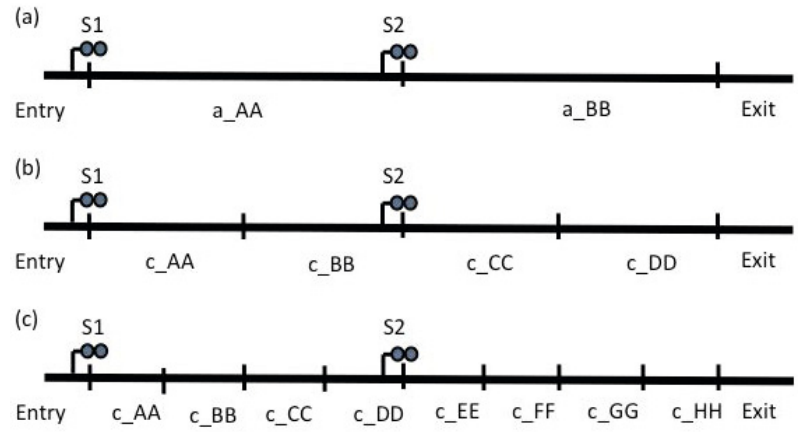
route (illustrated using 3

Fig. 3. Linear Scenario trains):

\begin{tabular}{|c|c|c|c|c|c|}
\hline number of tracks per abstract track & 1 & 2 & 4 & 8 & 16 \\
\hline number of states & 596 & 806 & 1472 & 3483 & 9615 \\
\hline
\end{tabular}

In the following, we develop and formalise an abstraction mechanism which reduces the number of tracks per route and thus gives an advantage in model checking. Figure 2 illustrates our abstraction: part (b) shows a concrete track plan to which part (a) is the abstract counterpart.

As discussed in Section 3.1. a track plan is essentially given by the set ALLTRACK of its track circuits and a relation next between them. We use the prefix $a_{-}$for abstract, and $c_{-}$for concrete when considering two track plans and the relationship between them. Thus, $a_{-} A L L T R A C K$ is the abstract set of track circuits (of tracks and points). Similarly, $c_{-} A L L T R A C K$ is the concrete set of track circuits. We assume that these are disjoint, apart from the special element nullTrack. The relations $a \_n e x t$ and $c \_n e x t$ define how track circuits are connected. Each concrete track circuit is associated with one abstract track circuit, defined by the following total surjective function:

$$
\text { abs : c_ALLTRACK } \gg \text { a_ALLTRACK }
$$

such that abs(nullTrack $)=$ nullTrack . 
The definition of $a b$ s for the one-station example is as follows in terms of relational image:

$$
\begin{aligned}
a b s\left[\left\{c_{-} T A A, c_{-} T A B, c_{-} T A C\right\}\right] & =\left\{a_{-} T A A\right\} \\
a b s\left[\left\{c_{-} T B A, c_{-} T B B, c_{-} T B C, c_{-} T B D\right\}\right] & =\left\{a_{-} T B A\right\} \\
a b s\left[\left\{c_{-} T A D, c_{-} T A E, c_{-} T A F, c_{-} T A G\right\}\right] & =\left\{a_{-} T A B\right\} \\
a b s\left[\left\{c_{-} T A H\right\}\right] & =\left\{a_{-} T A C\right\} \\
a b s\left[\left\{c_{-} T A I, c_{-} T A J\right\}\right] & =\left\{a_{-} T A D\right\}
\end{aligned}
$$

There are a number of necessary conditions on the abstraction function abs. These include prominently:

- Points are preserved under abstraction, i.e., a track circuit belonging to a point in the concrete topology is mapped to a point in the abstract topology.

- Routes are preserved under abstraction, e.g., $a b s\left[\left\{c_{-} T A D, c_{-} T A E, c_{-} T A F, c_{-} T A G, c_{-} T A H\right\}\right]$ cannot be $\left\{a_{-} T B A\right\}$ since the set of concrete track circuits is not within one route.

- Any concrete $c \_n e x t$ pair of track circuits should either both be related to the same abstract track circuit, or should reflect the relation between an abstract a_next pair, i.e.,

$$
\begin{aligned}
& \left.\forall c_{\_} t 1, c_{-} t 2 \bullet\left(c \_t 1 \mapsto c \_t 2\right) \in c_{-} n e x t\right) \Rightarrow \\
& \quad a b s\left(c \_t 1\right)=a b s\left(c \_t 2\right) \vee\left(a b s\left(c \_t 1\right) \mapsto a b s\left(c \_t 2\right)\right) \in a \_n e x t
\end{aligned}
$$

For example, a move within the same abstract track circuit is given by $\left(c_{-} T A B \mapsto c_{-} T A C\right) \in c_{-} n e x t \Rightarrow a b s\left(c_{-} T A B\right)=a b s\left(c_{-} T A C\right)$.

Beside the $a b s$ function, there are further functions needed in order to describe the full abstraction between track plans. They allow to formulate further conditions upon the relations defined in a track plan also on the tables, e.g.,

$$
\text { a_clearTable }{ }_{9} a b s^{-1}=c \_c l e a r T a b l e
$$

Our modelling approach works generically for all scheme plans. Thus, given a concrete and an abstract one, we have two formal models to compare. This comparison is performed using B refinement and CSP trace refinement. In the following, we focus on the B refinement.

We establish the refinement relationship between the Interlocking B machines by relating states with a linking invariant. To this end, we prove that each operation preserves the linking invariant. The linking invariant consists of three parts: the relationship between the positions of the trains $a_{-} p o s=c_{-}$pos ${ }_{9}^{\circ} a b s$, the relationship between the current positions of the points (which follows directly due to the static relationships), and the relationship between the track equipment which remains unchanged.

We illustrate the proof by comparing abstract and concrete versions of the move operation. For example, the concrete move.c_TAC.c_TAD corresponds to the abstract move.a.a_TAA.a_TAB; here, both have an effect on the B state. 
In contrast to this, the concrete move.a.c_TAB.c_TAC corresponds to the abstract move.a.a_TAA.a_TAA; the latter has no effect on the B state. Therefore, we can consider the abstract event move.a.a_TAA.a_TAA as the $\mathrm{B}$ operation skip. In a B refinement, a new concrete event can refine skip. This can be expressed in the following two lemmas:

Lemma 1 (Renamed move). If $\left(a b s\left(c \_t 1\right) \mapsto a b s\left(c \_t 2\right)\right) \in a \_n e x t$ then

$$
a b s\left(c \_t 1\right), a b s\left(c \_t 2\right) \longleftarrow a \_m o v e(t) \sqsubseteq c \_t 1, c \_t 2 \longleftarrow c \_m o v e(t)
$$

Lemma 2 (New move). If abs $\left(c_{-} t 1\right)=a b s\left(c_{-} t 2\right)$ then

$$
c \_t 1, c \_t 2 \longleftarrow \operatorname{skip}(t) \sqsubseteq c \_t 1, c \_t 2 \longleftarrow c_{-} \text {move }(t)
$$

As a consequence of the above lemmas (and similar lemmas for all other operations) the relationship between the abstract machine $M_{A}$ and the concrete one $M_{C}$ is given by $M_{A} \sqsubseteq_{T} f\left(M_{C} \backslash N\right)$, where $f$ and $N$ are defined by:

$$
\begin{aligned}
f(\text { move.a.currp.newp }) & =\text { move.a.abs (currp).abs }(\text { newp }) \\
N & =\{\text { move.a.currp.newp } \mid \text { abs }(\text { currp })=a b s(\text { newp })\}
\end{aligned}
$$

for all trains $a$ in the abstract and the concrete model.

Hence we can now consider the combination of the B machines $M_{A}$ and CSP processes $P_{A}$ to obtain:

Theorem 4. Let abs be an abstraction function from a concrete topology to an abstract topology. Let $P_{A} \| M_{A}$ be the CSP\|B model wrt the abstract topology, let $P_{C} \| M_{C}$ be the CSP\|B model wrt the concrete topology, such that both models are defined over the same set of trains. Let

1. $M_{A} \sqsubseteq_{T} f\left(M_{C} \backslash N\right)$ and

2. $P_{A} \sqsubseteq T f\left(P_{C} \backslash N\right)$.

Then collision (derailment) freedom of $P_{A} \| M_{A}$ implies collision (derailment) freedom of $P_{C} \| M_{C}$.

Proof. We compute:

$$
\begin{aligned}
P_{A} \| M_{A} & \left.\sqsubseteq_{T} f\left(P_{C} \backslash N\right) \| f\left(M_{C} \backslash N\right) \quad \text { (by conditions } 1 \text { and } 2\right) \\
& \sqsubseteq_{T} f\left(P_{C} \backslash N \| M_{C} \backslash N\right) \quad \text { (by distributivity of renaming) } \\
& \sqsubseteq_{T} f\left(\left(P_{C} \| M_{C}\right) \backslash N\right) \quad \text { (by distributivity of hiding) }
\end{aligned}
$$

With regards to collision freedom, we obtain:

$P_{A} \| M_{A}$ is collision free $\Rightarrow f\left(\left(P_{C} \| M_{C}\right) \backslash N\right)$ is collision free

(by trace refinement)

$$
\Leftrightarrow P_{C} \| M_{C} \backslash N \text { is collision free }
$$

(as $f($ collision $)=$ collision $)$

$\Leftrightarrow P_{C} \| M_{C}$ is collision free $\quad($ as collision $\notin N)$

Similarly for derailment freedom. 
Note that Theorem 4 decomposes the proof obligation into a B proof and a CSP proof respectively. In order to establish condition 1, we sketched above a general construction based upon techniques related to B refinement. Condition 2 can be verified using the model checker FDR on CSP processes only.

\section{Example Scenarios of CSP||B Railway Models}

In order to demonstrate the effectiveness of our techniques outlined in Section 4 and Section 5 we conducted experiments on five scenarios. The experiments were carried out using ProB 1.3.5 beta 15 [3] to verify the collision and derailment freedom of the abstract and concrete track plans using CTL model checking over the CSP ||B models. The number of trains involved is chosen according to the results of Section 4, collision freedom is checked with number of routes plus one train, derailment freedom is checked with one train. If the verification is successful then we conclude that the model is right and has the right properties. The CSP $\| \mathrm{B}$ models were also required to be divergence- and deadlock-free. Figure 4 summarises that all our scenarios are collision- and derailment-free.

To give an indication of the size of the track plans: scenario 1 has 6 tracks, 0 points, 2 signals and 2 routes; scenario 2 has 10 tracks, 0 points, 2 signals and 2 routes; scenario 3 has 16 tracks, 2 points, 3 signals and 4 routes; scenario 4 has 15 tracks, 1 point, 5 signals and 6 routes, and finally scenario 5 has 22 tracks, 2 points, 9 signals and 10 routes.

Notice that in all scenarios there is a significant reduction in the number of states being explored, comparing the abstract scenarios with the concrete scenarios. In order to achieve the desired verification results, however, abstraction is necessary only in scenario 4(b).

We gain full verification for the first four scenarios thanks to our two reduction techniques. Scenario 5 can be checked for derailment freedom, however, it cannot be checked for collision freedom. Thus, verification is only partial. However, we make the conjecture that it is possible to strengthen Theorem 1 rather than establishing collision freedom for number of routes plus one trains, it is sufficient to verify collision-freedom with two trains only. Figure 4 shows that verification of the double junction with two trains is possible. The double junction scenario is one which we have referred to in our previous work [13], it provides an interesting example of abstraction since the abstraction surrounding one of the points is a biased one, i.e., the normal position of one of the points remains unchanged in the abstraction, whereas the reverse position of the point is an abstraction of its track circuit and another track circuit. We will revisit the topic of abstractions when, in future work, we come to models which deal with bi-directional track circuits.

\section{Related Work}

Several industrial studies have been done on using model checking to verify railway applications, e.g., for example SNCF [2], and it is clear that their formal 


\begin{tabular}{|l|l|r|r|r|}
\hline Scenario & Model & $\begin{array}{r}\sharp \\
\text { Trains }\end{array}$ & $\begin{array}{r}\text { Abstract } \\
\text { States Checked }\end{array}$ & $\begin{array}{r}\text { Concrete } \\
\text { States Checked }\end{array}$ \\
\hline \hline 1 (a) derailment & $\begin{array}{l}\text { Linear with 2 tracks } \\
\text { per route }\end{array}$ & 1 & 27 & 31 \\
\hline 1(b) collisions & $\begin{array}{l}\text { Linear with 2 tracks } \\
\text { per route }\end{array}$ & 3 & 596 & 806 \\
\hline \hline 2(a) derailment & $\begin{array}{l}\text { Linear with 4 tracks } \\
\text { per route }\end{array}$ & 1 & 27 & 39 \\
\hline 2(b) collisions & $\begin{array}{l}\text { Linear with 4 tracks } \\
\text { per route }\end{array}$ & 3 & 596 & 1472 \\
\hline \hline $3(\mathrm{a})$ derailment & Station & 1 & 70 & 203 \\
\hline 3(b) collisions & Station & 5 & 151,508 & 968,700 \\
\hline \hline 4(a) derailment & Single Junction & 1 & 600 & 756 \\
\hline 4(b) collisions & Single Junction & 7 & 326,405 & Not completed \\
\hline \hline 5(a) derailment & Double Junction & 1 & 103,598 & 158,190 \\
\hline 5(b) collisions & Double Junction & 2 & 173,846 & 379,404 \\
\hline 5(c) collisions & Double Junction & 3 & Not completed & Not completed \\
\hline
\end{tabular}

Fig. 4. Variations of Five Example Scenarios checked

analysis is industrially important. To put our work into context we must first clarify that railway verification falls into two categories: the verification of railway designs prior to their implementation and the verification of the implementation descriptions themselves. Our work is in the first area. A comparison using different model checkers in the analysis of control tables has been conducted by Ferrari et al. [6] and falls into the first category. Winter in a recent paper [16] considers different optimising strategies for model checking using NuSMV and demonstrates the efficiency of their approach on very large models. These analyses also fall into the first category but the models are flat in structure compared to our models as they are defined in terms of boolean equations and do not focus on providing behavioural models. The analysis of interlocking tables (cf. control tables) by Haxthausen [7] also falls into the first category and is supported by automated tools that generate the models. The results achieved are comparable in size to our Single Junction scenario. Cimatti et al. [5] also have had considerable success using NuSMV but their analysis is focussed on the implementation descriptions.

Others have applied theorem proving in the verification of railway interlocking systems, for example, the Advance FP7 project [1] is developing Event-B models of such systems and verifying comparable safety properties. Indeed it would be interesting for us to investigate further the relationship between the combination of generic proofs and model cecking. In this paper, we have demonstrated that the data abstraction on the $\mathrm{B}$ part of the CSP $\| \mathrm{B}$ models is generic but more work will be needed on this when we enrich the models to contain trains which extend over more than one track circuit and can move in more than one direction. 
The research most closely related to ours is Winter [17. The way in which the ASM models are defined closely relates to ours since they have the same concept of routes, which contain tracks and points, between two signals, and contain a static and a behavioural definition. Their models are more advanced than ours since we currently restrict ourselves to have signals in one direction and we do not include shunting. The simplifications to the Winter models includes combining multiple track circuits into one provided they are always grouped together in the control table; this again resonates with the data abstraction we defined in Section 5, but we formalise the abstraction more explicitly.

\section{Conclusion}

We have successfully complemented our faithful modelling approach of railway interlockings as presented in 1312] by defining abstraction techniques that yield effective and efficient verification process based on model checking. We illustrated this process in terms of various scenarios. The correctness arguments in Sections 4 provides a new proof technique for event- and state-based reasoning. Section 5 demonstrates an interesting data abstraction using decomposition.

Heitmeyer in [8] discusses the importance of complete abstractions. Our abstractions are sound. It is future work to investigate if completeness can be established. In Section [6 we identified that the reduction of Theorem 1 is not sufficient for complex scheme plans. Here we hope to prove our conjecture that two trains are sufficient to verify collision freedom. Our current models lack certain details as discussed in Section 7. Adding these features will allow us to study more fine grained data abstractions. Following recent discussions with Winter, we also agree that another obvious optimisation to consider is the decomposition of track schemes.

Acknowledgement. The authors would like to thank S. Chadwick and D. Taylor from the company Invensys Rail for their support and encouraging feedback.

\section{References}

1. Advance FP7 project, http://www.advance-ict.eu/ (accessed: July 23, 2012)

2. Practical formal validation method for interlocking or automated systems, http://www.dcds11.uni-saarland.de/plenaries/practical-formalvalidation-method-for-interlocking-or-automated-systems.html (accessed: July 23, 2012)

3. ProB 1.3.5 beta15, http://www.stups.uni-duesseldorf.de/ProB (accessed: July 23, 2012)

4. Abrial, J.-R.: The B-Book: Assigning Programs to Meanings. CUP (1996)

5. Cimatti, A., Corvino, R., Lazzaro, A., Narasamdya, I., Rizzo, T., Roveri, M., Sanseviero, A., Tchaltsev, A.: Formal verification and validation of ERTMS industrial railway train spacing system. In: Madhusudan, P., Seshia, S.A. (eds.) CAV 2012. LNCS, vol. 7358, pp. 378-393. Springer, Heidelberg (2012) 
6. Ferrari, A., Magnani, G., Grasso, D., Fantechi, A.: Model checking interlocking control tables. In: FORMS/FORMAT, pp. 107-115 (2010)

7. Haxthausen, A.E.: Automated generation of safety requirements from railway interlocking tables. In: Margaria, T., Steffen, B. (eds.) ISoLA 2012, Part II. LNCS, vol. 7610, pp. 261-275. Springer, Heidelberg (2012)

8. Heitmeyer, C.L., Kirby, J., Labaw, B.G., Archer, M., Bharadwaj, R.: Using abstraction and model checking to detect safety violations in requirements specifications. IEEE Trans. Software Eng. 24(11), 927-948 (1998)

9. Hoare, C.A.R.: Communicating Sequential Processes. Prentice-Hall (1985)

10. Isobe, Y., Moller, F., Nguyen, H.N., Roggenbach, M.: Safety and line capacity in railways - an approach in Timed CSP. In: Derrick, J., Gnesi, S., Latella, D., Treharne, H. (eds.) IFM 2012. LNCS, vol. 7321, pp. 54-68. Springer, Heidelberg (2012)

11. Bjørner, D.: TRain: The Railway domain - A "Grand Challenge" for Computing Science \& Transportation Engineering. In: Jacquart, R. (ed.) Building the Information Society, IFIP 18th World Computer Congress, Topical Sessions, Toulouse, France, August 22-27, pp. 604-612. Kluwer (2004)

12. Moller, F., Nguyen, H.N., Roggenbach, M., Schneider, S., Treharne, H.: Combining event-based and state-based modelling for railway verification. Technical Report CS-12-02, University of Surrey (2012)

13. Moller, F., Nguyen, H.N., Roggenbach, M., Schneider, S., Treharne, H.: Railway modelling in CSP $\|$ B: the double junction case study. In: AVOCS (2012)

14. Morgan, C.C.: Of wp and CSP. In: Beauty Is Our Business: A Birthday Salute to Edsger J. Dijkstra. Springer (1990)

15. Schneider, S., Treharne, H.: CSP theorems for communicating B machines. Formal Asp. Comput. 17(4), 390-422 (2005)

16. Winter, K.: Optimising ordering strategies for symbolic model checking of railway interlockings. In: Margaria, T., Steffen, B. (eds.) ISoLA 2012, Part II. LNCS, vol. 7610, pp. 246-260. Springer, Heidelberg (2012)

17. Winter, K., Robinson, N.J.: Modelling large railway interlockings and model checking small ones. In: ACSC, pp. 309-316 (2003) 